\title{
ON THE NUMERICAL DETERMINATION OF OPTIMAL TEXTURES OF ALUMINIUM
}

\author{
P. BURGHOLZER ${ }^{1}$ AND O. SCHERZER ${ }^{2}$ \\ ${ }^{1} A M A G-F o r s c h u n g s-$ und Entwicklungsges.m.b.H., A-5282 Ranshofen, Austria, \\ now Institut für Theoretische Physik, Johannes Kepler Universität Linz, \\ A-4040 Linz, Austria \\ ${ }^{2}$ Christian-Doppler-Labor für Mathematische Modellierung und Numerische \\ Simulation, Institut für Mathematik, Johannes Kepler Universität Linz, \\ A-4040 Linz, Austria
}

In this paper a mathematical algorithm is studied to improve the deep-drawing quality of an aluminium sheet. The deep-drawing quality is usually expressed in terms of the normal anisotropie. In our mathematical model we use Taylor theory and ideal orientations to reformulate this problem as a nonlinear optimization problem for the normal anisotropie. Some numerical examples are presented.

KEY WORDS: Taylor theory, r-value, optimization, inverse problem.

\section{THE MODEL}

The deep-drawing quality of an aluminium sheet is usually expressed in terms of the normal anisotropie $r$ as a function of the angle $\alpha$ to the rolling direction of the aluminium sheet. Since during the tension test the volume of the aluminium specimen remains constant, we obtain in the stationary case for the strain rates

$$
\dot{E}_{11}+\dot{E}_{22}+\dot{E}_{33}=0 \text {, }
$$

where $\dot{E}_{i j}$ denotes the $(i, j)$-th component of the strain rate; the $z$-coordinate is used as the symmetry axis of the specimen. Since $\dot{E}_{11}$ is known in a tension test, we can rewrite the strain rates:

$$
\dot{E}=\dot{E}_{11}\left(\begin{array}{lll}
1 & 0 & 0 \\
0 & -q & 0 \\
0 & 0 & q-1
\end{array}\right) \text {. }
$$

We have the following relation between the normal anisotropie $r$ and $q$ :

$$
r=\frac{q}{1-q}
$$

Supported by the Christian Society, Austria. 
Mathematically the texture of aluminium is described by a density function $f(\psi)$, the orientation distribution function (ODF) [Bunge 1982]. $f(\psi)$ described the portion of the crystal with orientation $\psi$; every crystal can be transformed into a reference configuration by rotation via the Euler angle $\psi=\left(\phi_{1}, \Phi, \phi_{2}\right)$.

If we denote the work, necessary to deform a single crystal with orientation $\psi$ according to (1.2) (with $\left.\dot{E}_{11}=1\right)$ by $M(\alpha, q, \psi)$, we obtain for the total work, using the basic assumption of the Taylor Theory, that microscopic deformation behave like the macroscopic deformation:

$$
\bar{M}(\alpha, q)=\int M(\alpha, q, \psi) f(\psi) d \psi .
$$

While starting the tension test the strain ratio $q$ cannot be prescribed; due to the Principle of Minimal Work the number $q$ is adopted during the experiment, which minimizes the work, i.e., the work, which is performed during an experiment, is

$$
\min _{q} \bar{M}(\alpha, q) \text {; }
$$

here $\min _{q}$ symbolizes that $\bar{M}(\alpha, q)$ is minimized with respect to $q$, for fixed $\alpha$.

The value, which minimizes this functional, determines the contraction and strain ratios:

$$
q(\alpha):=\operatorname{argmin}_{q} \bar{M}(\alpha, q),
$$

i.e., $q(\alpha)$ denotes the number, where $\bar{M}(\alpha, q)$ is minimized.

We are interested in the computation of a physically relevant ODF $f$, for which the according values of $q(\alpha)$ satisfy additional assumptions, e.g. that $q(\alpha)$ becomes large; note that a large value of $q(\alpha)$ guarantees a large value of $r(\alpha)$, and a large value of $r(\alpha)$ during a tension test shows that the width changes more rapidly than the thickness of the specimen; which is a sign for a good quality of the aluminium sheet.

It is relatively simple to compute the contraction ratio (or strain ratio) for a given ODF, i.e. the input to output map; the reverse formulation, to recover from desired properties of the contraction ratio a physically relevant ODF is much more delicate:

1. From the desired properties of the contraction ratio we have to find a physically meaningful "mean Taylor factor" $\bar{M}(\alpha, q)$, i.e., we have to calculate $\bar{M}(\alpha, q)$ itself from the minimizing element $q(\alpha)$ of $\bar{M}(\alpha, q)$, what is only possible by applying physically relevant restrictions on the "mean Taylor factor", which are derived from physically relevant ODFs;

2. Using the "mean Taylor factor" we have to solve the unstable Fredholm integral equation of the first kind (1.4) to calculate the desired ODF.

The aim of this paper is to construct a numerical algorithm for solving the Inverse Problem, to find physically relevant ODFs of textures of aluminium which locally maximize the contraction ratio.

Physically relevant ODFs of textures of aluminium can be described via ideal orientations (see e.g. [Bunge 1970 Burgholzer 1993]), and each ideal orientation can be described by a Gauss density function

$$
f(\rho)=S_{0} e^{-\frac{\theta^{2}}{\theta_{0}^{2}}}
$$


where $\theta$ denotes the distance between $\rho$ and the Euler angle of the ideal orientation, $\theta_{0}$ denotes the dispersion and $\frac{1}{S_{0}}=\int e^{-\frac{\theta^{2}}{\theta_{0}^{2}}} d \rho$ (since $f$ is a density function). From the literature at least seven ideal orientations are known, [Bunge 1970] - and a few more, which are smeared over the $\alpha$ - and $\beta$-fibers [Burgholzer 1993]. For the moment we neglect effects of smeared ideal orientations; this could be the topic of further investigations. The Euler angles of the seven ideal orientations are summarized in Table 1. The texture of aluminium can be influenced during processing [Burgholzer 1993], and a different processing causes a different concentration of the ideal orientations.

The outline of this paper is as following:

in Section 2, we will remark some basic properties of the Euler angle representation of a crystal;

in Section 3, the description of a stable numerical algorithm will be given;

in Section 4, some numerical results will be presented.

\section{EULER ANGLES AND IDEAL ORIENTATIONS}

It is very common to describe the orientation of the crystals in an aluminium sheet by Euler angles. Although the Euler angles are very useful in treating the texture of aluminium, we have to be aware of the fact that the representation of a crystal by an Euler angle is not unique: Since aluminium is a face-centered cubic crystal it can be described by an orthonormal matrix $A$. The three line $\left(a_{11}, a_{12}, a_{13}\right),\left(a_{21}, a_{22}, a_{23}\right)$, $\left(a_{31}, a_{32}, a_{33}\right)$ of this matrix are orthonormal and describe the positions of the three axes of the crystal; the Euler angle of this crystal can be calculated from (cf. [Bunge 1970])

$$
\begin{aligned}
& a_{11}=\cos \left(\phi_{1}\right) \cos \left(\phi_{2}\right)-\sin \left(\phi_{1}\right) \sin \left(\phi_{2}\right) \cos (\Phi) \\
& a_{12}=\sin \left(\phi_{1}\right) \cos \left(\phi_{2}\right)+\cos \left(\phi_{1}\right) \sin \left(\phi_{2}\right) \cos (\Phi) \\
& a_{13}=\sin \left(\phi_{2}\right) \sin (\Phi) \\
& a_{21}=-\cos \left(\phi_{1}\right) \sin \left(\phi_{2}\right)-\sin \left(\phi_{1}\right) \cos \left(\phi_{2}\right) \cos (\Phi) \\
& a_{22}=-\sin \left(\phi_{1}\right) \sin \left(\phi_{2}\right)+\cos \left(\phi_{1}\right) \cos \left(\phi_{2}\right) \cos (\Phi) \\
& a_{23}=\cos \left(\phi_{2}\right) \sin (\Phi) \\
& a_{31}=\sin \left(\phi_{1}\right) \sin (\Phi) \\
& a_{32}=-\cos \left(\phi_{1}\right) \sin (\Phi) \\
& a_{33}=\cos (\Phi)
\end{aligned}
$$

\begin{tabular}{|c|c|c|c|c|c|}
\hline name & $\left(\begin{array}{lll}h & k & l\end{array}\right)$ & {$\left[\begin{array}{lll}u & v & w\end{array}\right]$} & $\phi_{1}$ & $\Phi$ & $\phi_{2}$ \\
\hline Cube & $\left(\begin{array}{lll}0 & 0 & 1\end{array}\right)$ & {$\left[\begin{array}{lll}1 & 0 & 0\end{array}\right]$} & $0^{\circ}$ & $0^{\circ}$ & $90^{\circ}$ \\
\hline CG & $\left(\begin{array}{lll}0 & 2 & 1\end{array}\right)$ & {$\left[\begin{array}{lll}1 & 0 & 0\end{array}\right]$} & $0^{\circ}$ & $26^{\circ}$ & $90^{\circ}$ \\
\hline Goss & $\left(\begin{array}{lll}0 & 1 & 1\end{array}\right)$ & {$\left[\begin{array}{lll}1 & 0 & 0\end{array}\right]$} & $0^{\circ}$ & $45^{\circ}$ & $90^{\circ}$ \\
\hline Brass & $\left(\begin{array}{lll}0 & 1 & 1\end{array}\right)$ & {$\left[\begin{array}{lll}2 & 1 & 1\end{array}\right]$} & $35^{\circ}$ & $45^{\circ}$ & $90^{\circ}$ \\
\hline$S_{3}$ & $\left(\begin{array}{lll}1 & 2 & 3\end{array}\right)$ & {$\left[\begin{array}{lll}6 & 3 & 4\end{array}\right]$} & $59^{\circ}$ & $37^{\circ}$ & $63^{\circ}$ \\
\hline Copper & $\left(\begin{array}{lll}1 & 1 & 2\end{array}\right)$ & {$\left[\begin{array}{lll}1 & 1 & 1\end{array}\right]$} & $90^{\circ}$ & $35^{\circ}$ & $45^{\circ}$ \\
\hline Taylor & $\left(\begin{array}{lll}4 & 4 & 11\end{array}\right)$ & {$\left[\begin{array}{lll}11 & 11 & 8\end{array}\right]$} & $90^{\circ}$ & $27^{\circ}$ & $45^{\circ}$ \\
\hline
\end{tabular}

Table 1 Ideal Orientations, Miller Indices and Euler Angles. 
It is of course clear that one describes the same crystal by interchanging the order of the unit-vectors or changing the signs of some of the unit-vectors; one should be aware that each Euler angle shown in Table 1 is only one representative of the crystal described by this Euler angle. Via symmetry considerations all possible representatives (in Euler angler) of a crystal can be calculated. From the seven ideal orientations all representatives are summarized in Table 2-8.

We defined the distance $\theta$ (in (1.6)) between $\left(h_{1}, H, h_{2}\right)$ and the ideal orientation $\left(\phi_{1}, \Phi, \phi_{2}\right)$ as the minimal angular distance, which is necessary to rotate the crystal $\left(h_{1}, H, h_{2}\right)$ into $\left(\phi_{1}, \Phi, \phi_{2}\right)$; to evaluate this distance, it is necessary to calculate all

Table 2 The Euler Angles of the Cube Orientation.

\begin{tabular}{ccccccccc}
\hline$\phi_{1}$ & $\Phi$ & $\phi_{2}$ & $\phi_{1}$ & $\Phi$ & $\phi_{2}$ & $\phi_{1}$ & $\Phi$ & $\phi_{2}$ \\
\hline $0^{\circ}$ & $90^{\circ}$ & $0^{\circ}$ & $0^{\circ}$ & $90^{\circ}$ & $90^{\circ}$ & $0^{\circ}$ & $90^{\circ}$ & $180^{\circ}$ \\
$90^{\circ}$ & $90^{\circ}$ & $0^{\circ}$ & $90^{\circ}$ & $90^{\circ}$ & $90^{\circ}$ & $90^{\circ}$ & $90^{\circ}$ & $180^{\circ}$ \\
$180^{\circ}$ & $90^{\circ}$ & $0^{\circ}$ & $180^{\circ}$ & $90^{\circ}$ & $90^{\circ}$ & $180^{\circ}$ & $90^{\circ}$ & $180^{\circ}$ \\
$a$ & $0^{\circ}$ & $90^{\circ}-a$ & $a$ & $0^{\circ}$ & $270^{\circ}-a$ & $b$ & $0^{\circ}$ & $180^{\circ}-b$ \\
$b$ & $0^{\circ}$ & $360^{\circ}-b$ & & & & & & \\
\hline
\end{tabular}

Table 3 The Euler Angles of the CG Orientation.

\begin{tabular}{ccccccccc}
\hline$\phi_{1}$ & $\Phi$ & $\phi_{2}$ & $\phi_{1}$ & $\Phi$ & $\phi_{2}$ & $\phi_{1}$ & $\Phi$ & $\phi_{2}$ \\
\hline $0^{\circ}$ & $26^{\circ}$ & $0^{\circ}$ & $0^{\circ}$ & $26^{\circ}$ & $90^{\circ}$ & $0^{\circ}$ & $26^{\circ}$ & $180^{\circ}$ \\
$0^{\circ}$ & $116^{\circ}$ & $0^{\circ}$ & $0^{\circ}$ & $116^{\circ}$ & $90^{\circ}$ & $0^{\circ}$ & $116^{\circ}$ & $180^{\circ}$ \\
$90^{\circ}$ & $90^{\circ}$ & $26^{\circ}$ & $90^{\circ}$ & $90^{\circ}$ & $64^{\circ}$ & $90^{\circ}$ & $90^{\circ}$ & $116^{\circ}$ \\
$90^{\circ}$ & $90^{\circ}$ & $154^{\circ}$ & $180^{\circ}$ & $154^{\circ}$ & $0^{\circ}$ & $180^{\circ}$ & $154^{\circ}$ & $90^{\circ}$ \\
$180^{\circ}$ & $154^{\circ}$ & $180^{\circ}$ & $180^{\circ}$ & $64^{\circ}$ & $0^{\circ}$ & $180^{\circ}$ & $64^{\circ}$ & $90^{\circ}$ \\
$180^{\circ}$ & $64^{\circ}$ & $180^{\circ}$ & & & & & & \\
\hline
\end{tabular}

Table 4 The Euler Angles of the Goss Orientation.

\begin{tabular}{ccccccccc}
\hline$\phi_{1}$ & $\Phi$ & $\phi_{2}$ & $\phi_{1}$ & $\Phi$ & $\phi_{2}$ & $\phi_{1}$ & $\Phi$ & $\phi_{2}$ \\
\hline $0^{\circ}$ & $45^{\circ}$ & $0^{\circ}$ & $0^{\circ}$ & $45^{\circ}$ & $90^{\circ}$ & $0^{\circ}$ & $45^{\circ}$ & $180^{\circ}$ \\
$0^{\circ}$ & $135^{\circ}$ & $0^{\circ}$ & $0^{\circ}$ & $135^{\circ}$ & $90^{\circ}$ & $0^{\circ}$ & $135^{\circ}$ & $180^{\circ}$ \\
$90^{\circ}$ & $90^{\circ}$ & $45^{\circ}$ & $90^{\circ}$ & $90^{\circ}$ & $135^{\circ}$ & $180^{\circ}$ & $135^{\circ}$ & $0^{\circ}$ \\
$180^{\circ}$ & $135^{\circ}$ & $90^{\circ}$ & $180^{\circ}$ & $135^{\circ}$ & $180^{\circ}$ & $180^{\circ}$ & $45^{\circ}$ & $0^{\circ}$ \\
$180^{\circ}$ & $45^{\circ}$ & $90^{\circ}$ & $180^{\circ}$ & $45^{\circ}$ & $180^{\circ}$ & & & \\
\hline
\end{tabular}

Table 5 The Euler Angles of the Brass Orientation.

\begin{tabular}{ccccccccc}
\hline$\phi_{1}$ & $\Phi$ & $\phi_{2}$ & $\phi_{1}$ & $\Phi$ & $\phi_{2}$ & $\phi_{1}$ & $\Phi$ & $\phi_{2}$ \\
\hline $35^{\circ}$ & $45^{\circ}$ & $0^{\circ}$ & $35^{\circ}$ & $45^{\circ}$ & $90^{\circ}$ & $35^{\circ}$ & $45^{\circ}$ & $180^{\circ}$ \\
$35^{\circ}$ & $135^{\circ}$ & $0^{\circ}$ & $35^{\circ}$ & $135^{\circ}$ & $90^{\circ}$ & $35^{\circ}$ & $135^{\circ}$ & $180^{\circ}$ \\
$55^{\circ}$ & $90^{\circ}$ & $45^{\circ}$ & $55^{\circ}$ & $90^{\circ}$ & $135^{\circ}$ & $125^{\circ}$ & $90^{\circ}$ & $45^{\circ}$ \\
$125^{\circ}$ & $90^{\circ}$ & $135^{\circ}$ & $145^{\circ}$ & $45^{\circ}$ & $0^{\circ}$ & $145^{\circ}$ & $45^{\circ}$ & $90^{\circ}$ \\
$145^{\circ}$ & $45^{\circ}$ & $180^{\circ}$ & $145^{\circ}$ & $135^{\circ}$ & $0^{\circ}$ & $145^{\circ}$ & $135^{\circ}$ & $90^{\circ}$ \\
$145^{\circ}$ & $135^{\circ}$ & $180^{\circ}$ & & & & & & \\
\hline
\end{tabular}


Table 6 The Euler Angles of the $S_{3}$ Orientation.

\begin{tabular}{ccccccccc}
\hline$\phi_{1}$ & $\Phi$ & $\phi_{2}$ & $\phi_{1}$ & $\Phi$ & $\phi_{2}$ & $\phi_{1}$ & $\Phi$ & $\phi_{2}$ \\
\hline $26.46^{\circ}$ & $122.43^{\circ}$ & $18.89^{\circ}$ & $26.46^{\circ}$ & $122.43^{\circ}$ & $71.11^{\circ}$ & $26.46^{\circ}$ & $122.43^{\circ}$ & $108.89^{\circ}$ \\
$26.46^{\circ}$ & $122.43^{\circ}$ & $161.11^{\circ}$ & $53.14^{\circ}$ & $74.14^{\circ}$ & $33.88^{\circ}$ & $53.14^{\circ}$ & $74.14^{\circ}$ & $56.12^{\circ}$ \\
$53.14^{\circ}$ & $74.14^{\circ}$ & $123.88^{\circ}$ & $53.14^{\circ}$ & $74.14^{\circ}$ & $146.12^{\circ}$ & $59^{\circ}$ & $37^{\circ}$ & $27^{\circ}$ \\
$59^{\circ}$ & $37^{\circ}$ & $63^{\circ}$ & $59^{\circ}$ & $37^{\circ}$ & $117^{\circ}$ & $5^{\circ}$ & $37^{\circ}$ & $153^{\circ}$ \\
$121^{\circ}$ & $143^{\circ}$ & $27^{\circ}$ & $121^{\circ}$ & $143^{\circ}$ & $63^{\circ}$ & $121^{\circ}$ & $143^{\circ}$ & $117^{\circ}$ \\
$121^{\circ}$ & $143^{\circ}$ & $153^{\circ}$ & $126.86^{\circ}$ & $105.86^{\circ}$ & $33.88^{\circ}$ & $126.86^{\circ}$ & $105.86^{\circ}$ & $56.12^{\circ}$ \\
$126.86^{\circ}$ & $105.86^{\circ}$ & $123.88^{\circ}$ & $126.86^{\circ}$ & $105.86^{\circ}$ & $146.12^{\circ}$ & $153.54^{\circ}$ & $57.57^{\circ}$ & $18.89^{\circ}$ \\
$153.54^{\circ}$ & $57.57^{\circ}$ & $71.11^{\circ}$ & $153.54^{\circ}$ & $57.57^{\circ}$ & $108.89^{\circ}$ & $153.54^{\circ}$ & $57.57^{\circ}$ & $161.11^{\circ}$ \\
\hline
\end{tabular}

Table 7 The Euler Angles of the Copper Orientation.

\begin{tabular}{ccccccccc}
\hline$\phi_{1}$ & $\Phi$ & $\phi_{2}$ & $\phi_{1}$ & $\Phi$ & $\phi_{2}$ & $\phi_{1}$ & $\Phi$ & $\phi_{2}$ \\
\hline $39.32^{\circ}$ & $66.07^{\circ}$ & $26.34^{\circ}$ & $39.32^{\circ}$ & $66.07^{\circ}$ & $63.66^{\circ}$ & $39.32^{\circ}$ & $66.07^{\circ}$ & $116.34^{\circ}$ \\
$39.32^{\circ}$ & $66.07^{\circ}$ & $153.66^{\circ}$ & $39.32^{\circ}$ & $113.93^{\circ}$ & $26.34^{\circ}$ & $39.32^{\circ}$ & $113.93^{\circ}$ & $63.66^{\circ}$ \\
$39.32^{\circ}$ & $113.93^{\circ}$ & $116.34^{\circ}$ & $39.32^{\circ}$ & $113.93^{\circ}$ & $153.66^{\circ}$ & $90^{\circ}$ & $35^{\circ}$ & $45^{\circ}$ \\
$90^{\circ}$ & $35^{\circ}$ & $135^{\circ}$ & $90^{\circ}$ & $145^{\circ}$ & $45^{\circ}$ & $9^{\circ}$ & $145^{\circ}$ & $135^{\circ}$ \\
$140.68^{\circ}$ & $66.07^{\circ}$ & $26.34^{\circ}$ & $140.68^{\circ}$ & $66.07^{\circ}$ & $63.66^{\circ}$ & $140.68^{\circ}$ & $66.07^{\circ}$ & $116.34^{\circ}$ \\
$140.68^{\circ}$ & $66.07^{\circ}$ & $153.66^{\circ}$ & $140.68^{\circ}$ & $113.93^{\circ}$ & $26.34^{\circ}$ & $140.68^{\circ}$ & $113.93^{\circ}$ & $63.66^{\circ}$ \\
$140.68^{\circ}$ & $113.93^{\circ}$ & $116.34^{\circ}$ & $140.68^{\circ}$ & $113.93^{\circ}$ & $153.66^{\circ}$ & & & \\
\hline
\end{tabular}

Table 8 The Euler Angles of the Taylor Orientation.

\begin{tabular}{ccccccccc}
\hline$\phi_{1}$ & $\Phi$ & $\phi_{2}$ & $\phi_{1}$ & $\Phi$ & $\phi_{2}$ & $\phi_{1}$ & $\Phi$ & $\phi_{2}$ \\
\hline $41.70^{\circ}$ & $71.28^{\circ}$ & $19.81^{\circ}$ & $41.70^{\circ}$ & $71.28^{\circ}$ & $70.19^{\circ}$ & $41.70^{\circ}$ & $71.28^{\circ}$ & $109.81^{\circ}$ \\
$41.70^{\circ}$ & $71.28^{\circ}$ & $160.19^{\circ}$ & $41.70^{\circ}$ & $108.72^{\circ}$ & $19.81^{\circ}$ & $41.70^{\circ}$ & $108.72^{\circ}$ & $70.19^{\circ}$ \\
$41.70^{\circ}$ & $108.72^{\circ}$ & $109.81^{\circ}$ & $41.70^{\circ}$ & $108.72^{\circ}$ & $160.19^{\circ}$ & $90^{\circ}$ & $27^{\circ}$ & $45^{\circ}$ \\
$9^{\circ}$ & $27^{\circ}$ & $135^{\circ}$ & $9^{\circ}$ & $153^{\circ}$ & $45^{\circ}$ & $9^{\circ}$ & $153^{\circ}$ & $135^{\circ}$ \\
$138.30^{\circ}$ & $71.28^{\circ}$ & $19.81^{\circ}$ & $138.30^{\circ}$ & $71.28^{\circ}$ & $70.19^{\circ}$ & $138.30^{\circ}$ & $71.28^{\circ}$ & $109.81^{\circ}$ \\
$138.30^{\circ}$ & $71.28^{\circ}$ & $160.19^{\circ}$ & $138.30^{\circ}$ & $108.72^{\circ}$ & $19.81^{\circ}$ & $138.30^{\circ}$ & $108.72^{\circ}$ & $70.19^{\circ}$ \\
$138.30^{\circ}$ & $108.72^{\circ}$ & $109.81^{\circ}$ & $138.20^{\circ}$ & $108.72^{\circ}$ & $160.19^{\circ}$ & & & \\
\hline
\end{tabular}

possible representatives of Euler angles of the crystals $\left(\phi_{1}, \Phi, \phi_{2}\right)$ and $\left(h_{1}, H, h_{2}\right)$. We denote the representatives of $\left(\phi_{1}, \Phi, \phi_{2}\right)$ by $\left(\phi_{1}(i), \Phi(i), \phi_{2}(i)\right), i=1, \ldots, n$ and the representatives of $\left(h_{1}, H, h_{2}\right)$ by $\left(h_{1}(j), H(j), h_{2}(j)\right), j=1, \ldots, m$. Then

$\theta=\min _{(i, j) \in\{1, \ldots, n\} \times\{1, \ldots, m\}} \sqrt{\left(h_{1}(i)-\phi_{1}(i)\right)^{2}+(H(j)-\Phi(j))^{2}+\left(h_{2}(j)-\phi_{2}(j)\right)^{2}}$.

We emphasize that in this paper we want to deal exemplarily with Ansatz-function (1.6), where the distance $\theta$ in (1.6) is chosen as in (2.1). Looking at the ODFs of Aluminium specimens it is well-known that the ODF is concentrated in only a few angles-the ideal orientations. As a function of the Euler angles an Ansatz-function for a realistic ODF should have peaks in the ideal orientations, with some dispersion around this peak (see e.g. [Bunge 1970]). A reasonable definition of distance between two crystals has to be independent of the representation of the crystals in Euler angles. At least, by our choice of Ansatz-functions these two basic requirements on an 
appropriate Ansatz-function for a physically relevant ODF are satisfied. The numerical algorithm described below uses as an input data the Ansatz-functions for the ideal orientations; for practical purpose even realistic data, obtained from experiments, could be used as Ansatz-functions - e.g.: If an Aluminium specimen is annealed, then the ODF almost looks like a Cube orientation; as an Ansatz-function in our algorithm for the Cube orientation even the measured data of the ODF of this annealed specimen could be used.

Moreover, we have compared our choice of Ansatz-functions with representations of the ideal orientations, which are used in the literature [Bunge 1991], and found that (1.6), (2.1) form an appropriate space of Ansatz-functions.

In the sequel, we will use the abbreviation $\int f(\rho) d \rho$ to symbolize that the function $f$ of the Euler angles $\rho$ is integrated over the whole space of Euler angles, and use the following relation (cf. [Bunge 1982]):

$$
f(\rho) d \rho=\frac{4}{\pi^{2}} \int_{0}^{\frac{\pi}{2}} \int_{0}^{\frac{\pi}{2}} \int_{0}^{\frac{\pi}{2}} f\left(\phi_{1}, \Phi, \phi_{2}\right) \sin (\Phi) d \phi_{1} d \Phi d \phi_{2} .
$$

\section{THE MATHEMATICAL MODEL}

As stated in Section 1, it is not possible in general to determine the mean Taylor factor from a given contraction ratio uniquely. Moreover, the calculation of an ODF from a mean Taylor factor is an unstable process. In order to calculate "optimal" ODFs in a stable way we characterize the set of all (physically relevant) attainable mean Taylor factors by a one to one correspondence between the ODF and its mean Taylor factor first. Using this approach we can cope with the nonuniqueness problem. Moreover, we can circumvent the unstable solution of the Fredholm integral equation of the first kind (1.4).

If we neglect the smearing of ideal orientations we obtain in a natural way a space of Ansatz-functions for the physically relevant orientation distribution functions, which we use in our numerical algorithm described below: The convex combination of seven Gauss-density functions, where each function describes an ideal orientation (see (1.6)).

For our further considerations we introduce a nonlinear mapping $F$, which maps our choice of physically relevant ODFs on the according mean Taylor factors:

$$
\left\{\begin{aligned}
F: D(F) & \rightarrow \mathbb{R} \\
\left(\eta_{i}, \theta_{0, i}\right)_{i=1}^{7} & \rightarrow \bar{M}(\alpha, q)
\end{aligned}\right.
$$

where $D(F):=\left\{\left(\eta_{i}, \theta_{0, i}\right)_{i=1}^{7}: \sum_{i=1}^{7} \eta_{i}=1, \theta_{0, i} \in\left[a_{i}, b_{i}\right]\right\} \subseteq \mathbb{R}_{2}^{7} ; \bar{M}(\alpha, q)$ denotes the mean Taylor factor according to the ODF $\sum_{i=1}^{7} \eta_{i} f_{i}\left(\theta_{0, i}\right)$, and $f_{i}\left(\theta_{0, i}\right)$ denotes the Gaussdensity function according to the $i$-th ideal orientation, with dispersion $\theta_{0, i}$ and $\eta_{i}$ denotes the intensity of the $i$-th ideal orientation; $\left[a_{i}, b_{i}\right]$ is the subset of $\left[0, \frac{\pi}{2}\right]$ containing the admissible dispersion of the $i$-th ideal orientation. The set $D(F)$ represents our choice of physically relevant ODFs.

Due to the fact that the function $F$ is linear in $\eta \in \mathbb{R}^{7}$, it is completely described by the values of $f_{i}\left(\theta_{0, i}\right)$. We calculated the mean Taylor factor for each Gauss-density 
function $f_{i}$ for the dispersion $\theta_{0, i}=2.5^{\circ}, 5^{\circ}, \ldots, 90^{\circ}, i=1, \ldots, 7$. The calculated values of $\bar{M}(\alpha, q):=\bar{M}\left(\alpha, q, \theta_{0, i}, i\right)$ where stored for each $\theta_{0, i} i$ on separate data-files. Thus we calculated $7 * 36$ data-files, where each file contains the 779 values of $\bar{M}(\alpha, q)$ $=\bar{M}\left(\alpha, q, \theta_{0, i}, i\right)$ for $\alpha=0^{\circ}, 5^{\circ}, \ldots, 90^{\circ}, q=0.025,0.05, \ldots, 1$.

As soon as these data files are calculated once a (locally) "optimal" ODF $f \in D(F)$ can be computed. For a given ODF $f=\sum_{i=1}^{7} \eta_{i} f_{i}\left(\theta_{0, i}\right)$ the according mean Taylor factor is given by

$$
\bar{M}(\alpha, q)=\sum_{i=1}^{7} \eta_{i} \bar{M}\left(\alpha, q, \theta_{0, i}, i\right) .
$$

Due to the Principle of Minimal Work we minimized (for fixed $\alpha$ ) the function $\bar{M}(\alpha, q)$ with respect to $q$ to calculate the contraction ratio $q(\alpha)$, i.e., the value $q$ which minimizes (for fixed $\alpha$ ) $\bar{M}(\alpha, q)$. The minimization of $\bar{M}(\alpha, q)$ can be done by several methods. We used the discrete values of $\bar{M}(\alpha, q)$ for $\alpha=0^{\circ}, 5^{\circ}, \ldots, 90^{\circ}$ and $q=$ $0,0.025,0.05, \ldots, 1$; the most simple way to compute the minimizer is to compare the (19) calculated values of the mean Taylor factor for fixed $\alpha$. This is the way the authors implemented this minimization. Another possible minimization would be to calculate appropriate spline-approximations for the values of the mean Taylor factor (for fixed $\alpha$ ); then the minimizer of this spline curve can be determined.

The maximization of the contraction ratio can be formulated as a constraint optimization problem

$$
\max _{D(F)} G\left(\left(\eta_{i}, \theta_{0, i}\right)_{i=1, \ldots, 7}\right)
$$

where $G\left(\left(\eta_{i}, \theta_{0, i}\right)_{i=1, \ldots, 7}\right)=\sum_{\alpha=0^{\circ}, 5^{\circ}, \ldots, 90^{\circ}} q^{2}(\alpha)$, and $q(\alpha)$ is the contraction ratio according to $\sum_{i=1}^{7} \eta_{i} f_{i}\left(\theta_{0, i}\right)$. We remark that this is a constraint optimization problem, where two types of constraints appear:

1. $\sum_{i=1}^{7} \eta_{i}=1$ (equality constraint),

2. $\theta_{0, i} \in\left[a_{i}, b_{i}\right]$ (inequality constraints).

It depends of the final aim, which objective function is used; instead of the objective

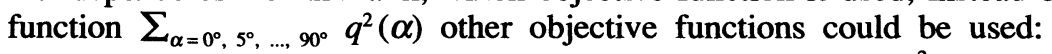

$$
-\sum_{\alpha=0^{\circ}, 5^{\circ}, \ldots, 90^{\circ}}\left(\frac{1}{19} \sum_{\alpha=0^{\circ}, 5^{\circ}, \ldots, 90^{\circ}} q(\alpha)-q(\beta)\right)^{2} .
$$

The aim of using this objective function would be to get a curve, where $q(\alpha)$ does not vary too much around its mid-value, i.e. that $q(\alpha)$ is isotropic.

\section{NUMERICAL RESULTS}

The numerical calculations have been made at the Abteilung Industriemathematik, Johannes Kepler Universität Linz, A-4040 Linz, Austria.

All numerical examples presented in this section were obtained on a VAXstation 4000. The main computational work in our algorithm is the computation of the datafiles in the first step of our algorithm. It took about 15 hours CPU-time on a VAXstation 4000. Nevertheless this computation has to be done only once. 
In the numerical algorithm, the second step is to find a local maximizer of the functional $G$ over $D(F)$ defined in (3.3) starting from a given initial guess. The mathematical search for a local maximizer of $G$ can be compared with the real experiment of making little changes of the adjustable parameters during the rolling process, while different global maxima may correspond to very different adjustments of the parameters during the deep drawing process.

For the first calculations shown below, we formulated the problem of minimizing $G$ as an unconstraint optimization problem. Roughly spoken, we extended the operator $G$ on the whole $\mathbb{R}_{2}^{7}$, by projecting every element of $\mathbb{R}_{2}^{7}$ onto $D(F)$. From numerical points of view it is unsatisfactory, to treat a constraint optimization problem as an unconstraint one, and in our future considerations we will treat the optimization problem as a constraint optimization problem. Then, the unconstraint optimization problem was solved by using a quasi-Newton method and a finite difference gradient, by using the subroutine DU MINF of the IMSL-Library [IMS].

Usually a nonlinear optimization problem cannot be solved globally. However, the functional $G$ depends linear on $\eta$ and only nonlinear on $\theta_{(o, i)}$, which reveals good possibilities to find the global extreme of the functional $G$, with a numerical algorithm.

Below we state several numerical examples. Each of the numerical examples consumed about 1 min CPU-time on a VAXstation 4000.

Example 4.1 We considered as initial guess: $100 \%$ Cube orientation with dispersion $\theta_{0, i}=17.5^{\circ}, i=1, \ldots, 7$. In our numerical algorithm we allowed to vary the dispersion between $7.5^{\circ}$ and $22.5^{\circ}$ and obtained $8 \%$ CG orientation, 24\% Goss orientation, $26 \%$ Brass orientation, $24 \% S_{3}$ orientation, $18 \%$ Copper orientation, all with dispersions $22.5^{\circ}$. The corresponding values of the contraction ratios are plotted in Figure 4.1. The value of the objective function $\sum_{i=1}^{18} q^{2}\left(\alpha_{i}\right)$ was increased from 1.79 for the initial guess to 9.37 for the optimized data by our numerical algorithm. We emphasize that the minimal value of $r(\alpha)$ (with respect to $\alpha$ ) was increased from 0.075 to 0.425 .

Example 4.2 We considered as initial guess: $25 \%$ Cube orientation, $35 \% S_{3}$ orientation, $40 \%$ Taylor orientation with dispersion $\theta_{0, i}=17.5^{\circ}, i=1, \ldots, 7$. In our numerical algorithm we allowed to vary the dispersion between $7.5^{\circ}$ and $22.5^{\circ}$ and obtained 2\% Cube orientation, 5\% CG orientation, 10\% Goss orientation, 29\% Brass orientation, $20 \% \mathrm{~S}_{3}$ orientation, $18 \%$ Copper orientation, $16 \%$ Taylor orientation, all with dispersions $22.5^{\circ}$. The corresponding values of the contraction ratios are plotted in Figure 4.2. The value of the objective function was increased from 5.7 for the initial guess to 9.0 for the optimized data by our numerical algorithm.

Example 4.3 We considered as same initial guess as in Example 4.1. But in contrast to this example, we considered now the objective function (3.4), to calculate an isotropic contraction ratio. We obtained $39 \%$ Cube orientation, with dispersion $22.5^{\circ}, 9 \%$ CG orientation, with dispersion $22.5^{\circ}, 2 \%$ Goss orientation, with dispersion $10.0^{\circ}, 13 \%$ Brass orientation, with dispersion $22.5^{\circ}, 14 \% S_{3}$ orientation, with dispersion $22.5^{\circ}, 17 \%$ Copper orientation, with dispersion $22.5^{\circ}, 7 \%$ Taylor orientation, with dispersion $22.5^{\circ}$. The corresponding values of the contraction ratios are plotted in Figure 4.3.

Example 4.4 We used the same initial guess as in Example 4.2, but used the objective function (3.4). We obtained 50\% Cube orientation, with dispersion $22.5^{\circ}$, 5\% CG orientation, with dispersion $7.5^{\circ}, 20 \% S_{3}$ orientation, with dispersion $12.5^{\circ}, 15 \%$ Copper orientation, with dispersion $10.0^{\circ}, 10 \%$ Taylor orientation, with dispersion $7.5^{\circ}$. The corresponding values of the contraction ratios are plotted in Figure 4.4. 


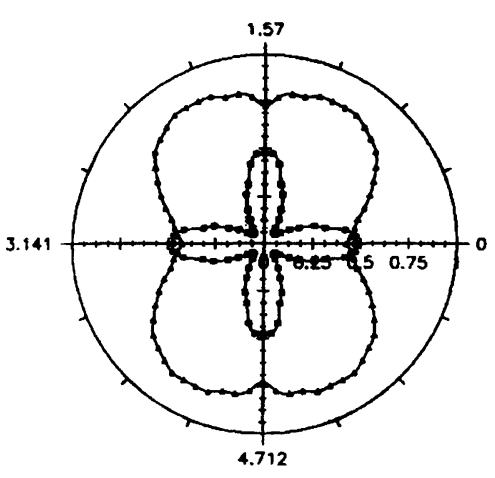

Figure 4.1 $\square: q(\alpha)$; initial guess, value of the objective function $\Sigma_{0^{\circ}, 5^{\circ}, \ldots, 90^{\circ}} q^{2}(\alpha)=1.8 ; \triangle$ : $q(\alpha)$; optimized values, value of the objective function $=9.4$.

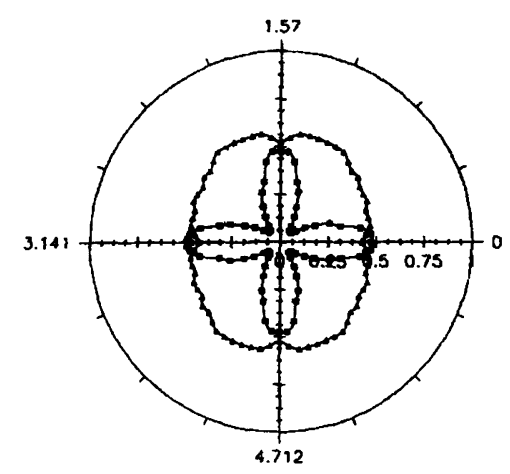

Figure 4.3 $\square: q(\alpha)$; initial guess, $\triangle: q(\alpha)$; optimized values; note: the objective function is now (3.4).

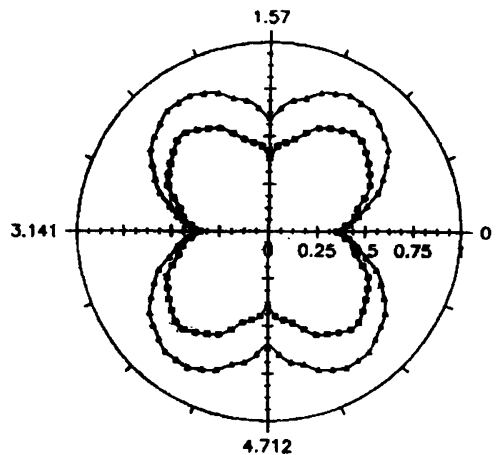

Figure 4.2 $\square: q(\alpha)$; initial guess, value of the objective function $\Sigma_{0^{\circ}, 5^{\circ}, \ldots, 90^{\circ}} q^{2}(\alpha)=5.7 ; \triangle$ : $q(\alpha)$; optimized values, value of the objective function $=9.0$.

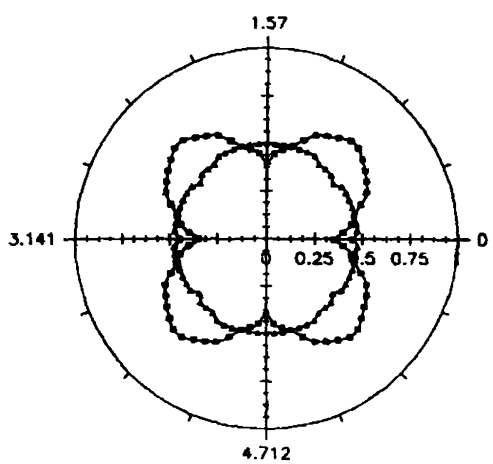

Figure $4.4 \square: q(\alpha)$; initial guess, $\triangle: q(\alpha)$; optimized values; note: the objective function is now (3.4).

Some critical comments on the numerical algorithm and further investigations

The mean Taylor factor depends nonlinearly on $\theta_{0, i}$, but for fixed $\theta_{0, i}$ the mean Taylor factor depends linearly on $\eta$ (cf. (3.2)). Moreover, the minimization of $\bar{M}(\alpha, q)$ with respect to $q$ (for fixed $\alpha$ ) is a nonlinear process. Usually for nonlinear optimization problems it is not possible to find global maximizers. This was investigated in our numerical examples too; starting from different initial guesses we found different (local) maximizers (compare Examples 4.1-4.4). The topic of future investigations will be to find global extrema.

The numerical examples above indicate that the contraction ratio can be influenced by the intensities of the ideal orientations. Moreover, the contraction ratio also depends on the dispersion of the ideal orientations. In the numerical examples it turned out large dispersion seem to be of advantage, for large contraction ratios. 


\section{Acknowledgements}

The authors wish to thank Prof. H. W. Engl (Universität Linz, Austria), Prof. Siegert (Universität Stuttgart, Germany), and Prof, U. M. Titulaer (Universität Linz, Austria) for fruitful discussions and their helpful advice; we also wish to thank Prof. H. J. Bunge (Universität Clausthal, Germany) for his very useful comments. Moreover, we express our thanks to AMAG-Forschungs- und Entwicklungsges.m.b.H., A-5282 Ranshofen, Austria for the permission to publish this manuscript.

\section{References}

1. Bunge, H. J. (1970). Some applications of the Taylor theory of polycrystal plasticity. Kristall und Technik, 5, 145-175.

2. Bunge, H. J. (1982). Texture Analysis in Material Science. Butterworths.

3. Bunge, H. J. (1991). Representations and interpretation of orientation distribution functions. In Bunge, H. J. and Esling, C., editor, Advances and Applications of Quantitative Texture Analysis, pages 19-48. DGM Informationsgesellschaft Verlag, Oberursel (FRG).

4. Burgholzer, P. (1993). Textur und plastische Anisotropie von Aluminiumblechen. $\mathrm{PhD}$ thesis, Universität Linz.

5. IMSL, 2500 ParkWest Tower One, 2500 CityWest Boulevard, Houston, Texas 77042-3020, USA. MATH/LIBRARY. FORTRAN Subroutines for Mathematical Applications. 